\title{
Quorum-Based Secure Multi-party Computation
}

\author{
Donald Beaver ${ }^{1}$ and Avishai Wool ${ }^{2}$ \\ 1 IBM/Transarc; beaver@transarc.com \\ 2 Bell Laboratories, Lucent Technologies; yash@research.bell-labs.com
}

\begin{abstract}
This paper describes efficient protocols for multi-party computations that are information-theoretically secure against passive attacks. The results presented here apply to access structures based on quorum systems, which are collections of sets enjoying a naturallymotivated self-intersection property. Quorum-based access structures include threshold systems but are far richer and more general, and they have specific applicability to several problems in distributed control and management. The achievable limits of security in quorum-based multiparty computation are shown to be equivalent to those determined by Hirt and Maurer in [HM97], drawing a natural but non-obvious connection between quorum systems and the extremes of secure multi-party computation. Moreover, for both the general case and for specific applications, the protocols presented here are simpler and more efficient.
\end{abstract}

\section{Introduction}

\subsection{Overview}

Multi-party computation - a means for groups to engage in joint computation as though an absolutely trusted third party were available to help them - has enjoyed a great deal of attention for several years, with much effort spent on exploring the limits of robustness and efficiency [GMW86, GMW87, GHY88, BGW88, CCD88, RB89, Bea91b, BG89, BH92, DDFY94, CFGN96]. Virtually without exception, all solutions have been based on threshold secret sharing, and hence they are themselves threshold-oriented. Recently, Hirt and Maurer characterized tolerable adversary sets in general multi-party computations without restriction to purely threshold-based sets [HM97]. Our work explores and expands on a non-threshold-based approach to multi-party computation, investigating quorum-based multi-party protocols and providing more efficient solutions for general and specific cases.

Whom to Trust. The general motivation for multi-party computation is simple: apply a decentralized approach to ensure robustness and security in joint computations, increasing reliability by dissipating trust among many individuals. Formally, a collection of $n$ players wish to compute some function $f\left(x_{1}, \ldots, x_{n}\right)$ of their respective, private inputs, revealing the final result but nothing more. Ideally, they could resort to a trusted third party to collect the inputs and return

\footnotetext{
${ }^{0}$ Part of this work was done at the DIMACS Research \& Education Institute Cryptography and Network Security Workshop (DREI'97), August 1997.
} 
only the result; the goal of secure multi-party computation is to achieve the same task without the existence of any such helper.

Threshold Schemes. Multi-party protocols are typically based on secret sharing. In the past virtually all of them relied on some form of Shamir's polynomialbased sharing scheme [Sha79, Bla79]. Because these sharing schemes are threshold based - i.e., reconstruction of a secret depends on having some minimal number of shares, rather than some particular set of players - there is a natural limit on the types of dishonest coalitions that can be tolerated by multi-party computations. In particular, an honest majority is necessary and sufficient for verifiable sharing and multi-party computation [RB89, Bea91b] - for threshold-based protocols. Conversely, as long as no dishonest coalition exceeds the threshold of size $\left\lfloor\frac{n-1}{2}\right\rfloor$, secure multi-party computation is achievable. This characterization of tolerable adversaries is natural, elegant, and seemingly all that needs be said.

Weighted Threshold Schemes. The simplest generalization involves a weighted approach, in which important players (such as CEO's and VP's) are essentially granted more shares of the secret than the peons. The characterization of tolerable adversaries remains simple; the weighted collection of shares held by honest players (resp. dishonest players) must be a majority (resp. minority) of all shares. Thus the characterization of what is achievable needs only slight modification. A disadvantage of such a weighted approach is that the important players have more shares so they need to perform more of the computational work.

Quorum Systems. The approach we take here is motivated by distributed control and management problems such as mutual exclusion (cf. [GB85]), data replication protocols (cf. [DGS85, Her84]) and name servers (cf. [MV88]). In these applications certain operations are allowed only if they are authorized by a set of members which is defined to be a quorum. The requirement from the quorum sets is that every two quorums should have at least one member in common. The existence of a common member helps prevent uncoordinated action between two different groups, whether that action be committing to a newly-written value or returning the latest version. Clearly, any threshold system (weighted or not) whose threshold exceeds half the total population (or weight) qualifies as a quorum system: the pigeonhole principle ensures that any two threshold-passing sets will intersect nontrivially.

Adding Security. Secret sharing schemes for arbitrary access structures have been developed by Ito, Saito, and Nishizeki [ISN87]. Schemes for structures represented by monotone formulas were proposed by Benaloh and Leichter [BL88]. More recently, Naor and Wool have designed schemes for structures which represent specific quorum systems [NW96]. The natural question to ask is whether these sharing schemes are usable for multi-party computations as well. We answer this question in the affirmative, and present several new protocols that use quorum-based secret sharing schemes. In the process we also present some new quorum secret-sharing schemes which we utilize in the protocols. Thus we are able to support non-threshold access structures without resorting to weighted threshold schemes. This allows us to escape the "honest majority" barrier to 
some degree - our protocols can tolerate some dishonest majorities of players. However, this comes at a price: the protocols cannot tolerate certain dishonest minorities.

Attack Model. In this work we focus on passive attacks, in which all the players follow the protocol. However, dishonest players may pool their information in order to learn some of the secret inputs of other players (this is sometimes called the "honest but curious" model). The adversary is only permitted to choose a dishonest coalition from the collection of coalitions that the protocol tolerates (these will be the complements of quorum sets). On the other hand, our security requirement is of the strongest type: that a dishonest coalition can learn nothing about the other's secret inputs, in the information-theoretic sense.

Efficiency. Once the security of a protocol has been established, we are interested in two measures of its quality: the number of messages sent per multiplication, which captures the communication complexity of the protocol; and the size of the shares each player holds, which captures the amount of local computation each player needs to perform.

\subsection{Connecting multi-party computation and quorum systems}

Multi-party computation protocols have traditionally been defined and analyzed in terms of the collection $\mathcal{B}$ of bad coalitions that they tolerate. For instance, for a threshold-based protocol this collection is of the form "all the sets of cardinality less than $t . "$ Early on it was discovered that there is a simple combinatorial condition which precludes the existence of any protocol that tolerates certain types of $\mathcal{B}$ 's. The following lemma has been proved in several places [BGW88, BG89, CK91] and follows from a direct reduction to two-party protocols.

Lemma 1. $A$ set system $\mathcal{B}$ is said to 2-cover the universe $U$ if two sets $B_{1}, B_{2} \epsilon$ $\mathcal{B}$ exist such that $B_{1} \cup B_{2}=U$. If $\mathcal{B}$ 2-covers $U$, then no multi-party protocol that tolerates $\mathcal{B}$ exists for computing the $A N D$ (or OR, or finite-field multiplication) function.

Recently Hirt and Maurer showed that the converse of Lemma 1 is also true (for honest-but-curious attacks), by describing a general protocol which tolerates any collection $\mathcal{B}$ that does not 2-cover $U$. Their protocol involves a recursive decomposition of the bad collection $\mathcal{B}$ into 3 sub-collections, and simulating the 2-of-3 threshold-based protocol of [BGW88] at each level of the recursion. Thus they proved the following characterization.

Theorem 2. [HM97] A multi-party protocol that tolerates $\mathcal{B}$ exists iff $U$ is not 2-covered by $\mathcal{B}$.

The connection between this characterization and our work is captured by the following observation.

Lemma 3. Let $\mathcal{B}$ be a collection of bad coalitions over $U$. Let $\mathcal{G}=\{G: U \backslash G \in$ $\mathcal{B}\}$ be the collection of complements of the bad coalitions. Then $\mathcal{B}$ does not 2-cover $U$ iff $\mathcal{G}$ is a quorum system. 
Proof: Consider some $B_{1}, B_{2} \in \mathcal{B}$, and let $G_{1}, G_{2} \in \mathcal{G}$ be the complements of $B_{1}, B_{2}$, respectively. If $u \notin B_{1} \cup B_{2}$ then clearly $u \in G_{1} \cap G_{2}$, and vice versa. Therefore every two sets of $\mathcal{B}$ do not cover $U$ iff every two sets of $\mathcal{G}$ have a non-empty intersection.

Therefore multi-party protocols are characterized equivalently by the quorum systems that they respect and by the bad coalitions they tolerate. However, focusing on the positive, quorum-based view leads naturally to the use of quorum secret sharing schemes, which form the foundation for our new protocols.

\subsection{Contributions}

Our first result is a new secure multi-party protocol which respects any arbitrary quorum system (and hence tolerates any collection of bad coalitions that does not 2-cover $U$ ). By this we show an alternative proof to the characterization of Hirt and Maurer [HM97]. However our protocol is much more efficient; for a system of $n$ players and $m$ minimal quorums our protocol sends $O\left(n^{2} m\right)$ messages per multiplication, as compared to the $\Omega\left(\mathrm{m}^{2.709}\right)$ messages sent by Hirt and Maurer's protocol. ${ }^{3}$ Note that in general $m=2^{\Omega(n)}$, so it is of far greater importance to reduce the dependence on $m$. Moreover, our protocol is much simpler to describe and to analyze, since it involves neither recursion nor simulation of subprotocols.

We also describe several multi-party protocols which are based on specific quorum systems. The quorum systems that we consider were devised for various distributed control mechanisms and are shown (in the referenced papers) to have favorable properties such as high availability and low load [NW94]. The quorum systems we consider are the crumbling wall quorum systems of [PW97], the finite projective plane (FPP) quorum system of [Mae85], and the 2-of-3 majority-tree systems of [AE91] and [Kum91]. The protocols we provide for these systems are all fully polynomial in $n$, with a complexity of $O\left(n^{2}\right)$ messages per multiplication. Thus to our knowledge they are the first non-threshold protocols which are as efficient as the original protocol of [BGW88, CCD88].

For the crumbling walls and tree-based systems we use the secret sharing schemes proposed in [NW96]. In the former scheme the shares are twice the size of the secrets, while the latter is optimal (i.e., the size of the shares equals the size of the secret). For the FPP system we present a new optimal secret sharing scheme, which may be interesting in its own right. Thus the local computations that the players perform in all these protocols are extremely efficient, typically consisting of a constant number of multiplications and a linear number of additions and random bit generations.

Organization: In $\S 2$ we define quorum systems, secret scharing schemes, and secure multi-party computation. In $\S 3$ we present our general quorum-based protocol for arbitrary quorum systems. $\S 4$ describes our efficient (polynomial in $n$ ) protocols for special quorum systems.

${ }^{3}$ In their paper Hirt and Maurer only claim that their protocol is polynomial in $m$. We provide a more detailed complexity analysis for their algorithm in the sequel. 


\section{Definitions}

\subsection{Quorum systems and access specifications}

Definition 4. Let $U$ denote a universe of players. A set system $\mathcal{Q}=$ $\left\{Q_{1}, \ldots, Q_{m}\right\}$ is a collection of subsets $Q_{i} \subseteq U$. A quorum system is a set system $\mathcal{Q}$ that has the intersection property: $Q_{i} \cap Q_{j} \neq \emptyset$ for all $Q_{i}, Q_{j} \in \mathcal{Q}$. The sets of the system are called quorums.

We use $n=|U|$ to denote the number of players. Unless otherwise noted, the quorums in all the quorum systems mentioned in this paper are minimal: $Q_{i} \nsubseteq$ $Q_{j}$ for every two quorums $Q_{i}, Q_{j} \in \mathcal{Q}$. We use $m=|\mathcal{Q}|$ to denote the number of (minimal) quorums.

Definition 5. An access specification is a disjoint pair $(\mathcal{G}, \mathcal{B})$ of collections of subsets, such that $\mathcal{G}$ is monotone increasing (that is, $\left(\forall G_{1} \in \mathcal{G}\right)\left(G_{1} \subseteq G_{2} \Rightarrow\right.$ $\left.G_{2} \in \mathcal{G}\right)$ ), and $\mathcal{B}$ is monotone decreasing.

Remark: The first collection, $\mathcal{G}$, describes sets of players that are permitted to access secrets; the second collection, $\mathcal{B}$, describes coalitions who should not learn anything about such secrets. Sets in $\mathcal{G}$ are called good, while those in $\mathcal{B}$ are called bad. Note that $(\mathcal{G}, \mathcal{B})$ need not be a partition; there may be sets that are neither good nor bad. If, however, $(\mathcal{G}, \mathcal{B})$ is a partition, namely $\mathcal{G} \cup \mathcal{B}=2^{U}$, then we say it is unambiguous.

Definition 6. Let $\mathcal{Q}$ be a quorum system. Let $\mathcal{G}(\mathcal{Q})=\{G \supseteq Q: Q \in \mathcal{Q}\}$ be the collection of sets containing some quorum, and let $\mathcal{B}(\mathcal{Q})=\{B: U \backslash B \in \mathcal{G}(\mathcal{Q})\}$ be the collection of sets whose complement contains a quorum. Then the quorum access specification of $\mathcal{Q}$ is $(\mathcal{G}(\mathcal{Q}), \mathcal{B}(\mathcal{Q})$ ).

Remark: Quorum systems whose access specification is unambiguous have many interesting properties. Such systems have attracted attention in several contexts, and much is known about them. The terminology associated with these systems differs from discipline to discipline, and a non-comprehensive list of names includes "non-dominated coterie" [GB85]; "simple decisive game" [Owe82]; "self-dual monotone boolean function" [IK93]; "ultrafilter" [BKK94]; and " $\nu$-critical hypergraph" [Für88].

\subsection{Secret sharing}

Definition 7. Let $U=\{1, \ldots, n\}$ and let $S$ be a finite set of secrets. A secretsharing scheme (SSS) is a mapping $\Pi: S \times R \mapsto S_{1} \times \cdots \times S_{n}$, where $R$ is a set of random strings, and for each $i \in U, S_{\imath}$ is a set of secret shares. $\Pi$ is said to realize an access specification $(\mathcal{G}, \mathcal{B})$ if it satisfies the following conditions:

1. The secret can be reconstructed by any subset in $\mathcal{G}$. That is, associated with every set $G \in \mathcal{G}\left(G=\left\{i_{1}, \ldots, i_{|G|}\right\}\right)$ there is a function $h_{G}: S_{i_{1}} \times \cdots \times$ $S_{i_{|G|}} \mapsto S$ such that for every $(s, r) \in S \times R$, if $\Pi(s, r)=\left\{s_{1}, \ldots, s_{n}\right\}$ then $h_{G}\left(s_{u_{1}}, \ldots, s_{v_{|G|}}\right)=s$. 
2. No subset in $\mathcal{B}$ can reveal any partial information about the secret (in the information theoretic sense). Formally, for any subset $B \in \mathcal{B}$, for every two secrets $a, b \in S$, and for every possible collection of shares $\left\{s_{i}\right\}_{i \in B}$ : $\operatorname{Pr}\left(\left\{s_{i}\right\}_{i \in B} \mid a\right)=\operatorname{Pr}\left(\left\{s_{i}\right\}_{i \in B} \mid b\right)$, where the probability is taken over the random string $r$.

\subsection{Multi-party computation}

The following formalizations are standard and based on commonly accepted approaches [GMR89, BGW88, CCD88, Bea91a, MR91]. Let $C$ be a circuit over logical (or finite arithmetical) gates, having $n$ inputs and one output, ${ }^{4}$ and let $f\left(x_{1}, \ldots, x_{n}\right)$ be the function it computes. A multi-party protocol is a set of $n$ interactive, probabilistic Turing machines, called players. A protocol computes $f$ if each player outputs $f\left(x_{1}, \ldots, x_{n}\right)$ at the end of execution.

An adversary class is a collection of subsets of $U=\{1, \ldots, n\}$. (Because we generally discuss the passive case, we identify adversaries with sets of observed players.) A partial $S$-view of an execution is the set of strings describing internal computations, random tosses, and input-output transcripts for players in $S$, up to some given round of interaction. We often overload the term view to mean a specific string in a given execution, the distribution on strings over all executions, or the partial view at some moment, as convenience dictates. An execution of a protocol is characterized by a result-vector of $n$ outputs along with the final view of the adversary.

Let simulator $S$ be given an adversary $B$ as input and have access to an ideal protocol in which a trusted, external party collects the inputs $x_{1}, \ldots, x_{n}$ and returns the result, $f\left(x_{1}, \ldots, x_{n}\right)$. In addition to providing progressive partial $B$-views, the simulator produces a final result-vector (whose adversary-view is the concatenation of the progressive partial $B$-views).

Definition 8. A protocol that computes $f$ is said to tolerate adversary class $\mathcal{B}$ if there is a simulator $S$ such that for any $B \in \mathcal{B}$ and any $x_{1}, \ldots, x_{n}$, the resultvector produced by $S$ is identically distributed to that obtained by executing the protocol. (The protocol is also said to tolerate each set $B \in \mathcal{B}$.)

Our constructions follow the conventional share-compute-reconstruct paradigm introduced in [GMW86]. Thus we have additional properties to seek, including whether particular sets are capable of reconstructing the final result.

Definition 9. A multi-party protocol for $f$ is said to respect a set $G$ if at the end of the computation phase, the members of $G$ can collectively reconstruct the function value. A protocol is said to respect a collection of sets if it respects each set in the collection.

Definition 10. A protocol is said to securely implement an access specification $(\mathcal{G}, \mathcal{B})$ if it tolerates $\mathcal{B}$ and respects $\mathcal{G}$.

Definition 11. Let $\mathcal{Q}$ be a quorum system. A protocol is said to be $\mathcal{Q}$-private if it securely implements the quorum access specification $(\mathcal{G}(\mathcal{Q}), \mathcal{B}(\mathcal{Q})$ ).

\footnotetext{
${ }^{4}$ This is easily generalized to multiple and private outputs.
} 
The preceding commentary is easily generalized to families of functions. A finite set of protocols is complete for some function family if it enables sharing and reconstruction, and through finite composition can evaluate any finite circuit securely. Our approach is standard [GMW86, BGW88, CCD88]: to provide efficient protocols for sharing, reconstruction, addition (linear combination), and multiplication.

\section{A general protocol}

In this section we present a general multi-party protocol which is $\mathcal{Q}$-private for any quorum system $\mathcal{Q}$. Let $\mathcal{Q}$ be a quorum system, and let $C$ be a circuit which computes a function $f$. To describe a $\mathcal{Q}$-private protocol which computes $f$, we need first to show a secret sharing scheme that realizes $(\mathcal{G}(\mathcal{Q}), \mathcal{B}(\mathcal{Q})$ ). Then it suffices to show how the players can compute sums and products $\mathcal{Q}$-privately using the shares. The computation of $f$ is performed by simulating the circuit $C$ gate by gate.

\subsection{The Gen-SSS secret sharing scheme}

Consider a quorum system $\mathcal{Q}=\left\{Q_{1}, \ldots, Q_{m}\right\}$ of minimal quorums, and let $x$ be the value to be shared. First $x$ is represented randomly as a sum-share $x=\sum_{j=1}^{m} x_{j}$. This can be done by assigning uniformly chosen random values to $x_{j}$ for $j=1, \ldots, m-1$ and assigning $x_{m}=x-\sum_{j=1}^{m-1} x_{j}$. We call the values $x_{1}, \ldots, x_{m}$ the parts of $x$ to differentiate them from the shares of the scheme. The share $s_{u}(x)$ of player $u$ is the set of parts corresponding to the quorums that contain $u$ :

$$
s_{u}(x) \leftarrow\left\{x_{j}\right\}_{Q_{j} \ni u} .
$$

Proposition 12. Gen-SSS is a secret sharing scheme realizing the quorum access specification $(\mathcal{G}(\mathcal{Q}), \mathcal{B}(\mathcal{Q})$ ).

Proof: Consider some set $G \in \mathcal{G}(\mathcal{Q})$ which contains the quorum $Q_{i} \in \mathcal{Q}$. By the intersection property, $Q_{i} \cap Q_{j} \neq \emptyset$ for all $j=1, \ldots, m$, so for every quorum $Q_{j}$ there exists a player $u \in Q_{i}$ which has the corresponding part $x_{j}$. Therefore the players in $Q_{i}$ collectively have all the parts $\left\{x_{j}\right\}_{j=1}^{m}$ and can reconstruct the secret $x$.

Now consider a set $B \in \mathcal{B}(\mathcal{Q})$. Then by definition there exists some quorum $Q_{i} \in \mathcal{Q}$ such that $Q_{i} \subseteq U \backslash B$. Only members of $Q_{\imath}$ receive the part $x_{i}$, and therefore no player in $B$ has $x_{i}$.

Let $\xi^{x}$ be a specific assignment of Gen-SSS shares which encodes a secret value $x$. Then for any other possible value $y$ there exists a secret-sharing $\xi^{y}$, which encodes $y$, such that the projections of $\xi^{x}$ and $\xi^{y}$ on the set $B$ are identical. $\xi^{y}$ is constructed from $\xi^{x}$ by replacing the part $x_{i}$ by $x_{i}+(y-x)$, and thus the change only affects the shares held by members of $Q_{i}$. Therefore

$$
\operatorname{Pr}\left(\left\{s_{u}(x)\right\}_{u \in B} \mid x\right)=\operatorname{Pr}\left(\left\{s_{u}(y)\right\}_{u \in B} \mid y\right),
$$

so the union of the shares of all the players in $B$ gives no information about $x$. 


\subsection{The Gen-MP protocol}

The Gen-MP protocol for evaluating a circuit obliviously is the direct composition of protocols for linear combination and multiplication, described below.

Computing a linear combination Assume that two secrets $x=\sum_{i=j}^{m} x_{j}$ and $y=\sum_{\imath=j}^{m} y_{j}$ are shared among the players according to the Gen-SSS scheme. Let $\alpha$ and $\beta$ be fixed constants. The players represent $z=\alpha x+\beta y$ as $z=\sum_{j=1}^{m} z_{j}$ as follows. Each player $u$ locally computes

$$
s_{u}(z) \leftarrow\left\{z_{j} \leftarrow \alpha x_{j}+\beta y_{j}\right\}_{Q_{j} \ni u} .
$$

It is easy to see that the new $s_{u}(z)$ shares are valid Gen-SSS shares for $z$, and since the computation did not involve the exchange of any messages the computation is $\mathcal{Q}$-private.

Computing a product As before let $x=\sum_{j=1}^{m} x_{j}$ and $y=\sum_{j=1}^{m} y_{j}$ be shared among the players according to Gen-SSS. The players aim to compute the product $z=x \cdot y=\sum_{i=1}^{m} \sum_{j=1}^{m} x_{i} y_{j}$.

The protocol depends on a mapping $\rho:[1 \ldots m] \times[1 \ldots m] \mapsto U$, for which $\rho(i, j) \in\left(Q_{i} \cap Q_{j}\right)$. Such a mapping $\rho$ exists since by the intersection property we have $\left(Q_{i} \cap Q_{j}\right) \neq \emptyset$. We say that a player $u$ is in charge of computing the term $x_{i} y_{j}$ if $\rho(i, j)=u$. In order to compute the product, each player $u$ performs the following steps:

1. Player $u$ locally computes the sum of all the terms she is in charge of: $w_{u} \leftarrow \sum_{i, j: \rho(i, j)=u} x_{i} y_{j}$.

2. Player $u$ secret-shares the value $w_{u}$ among all the players using the GenSSS scheme. Namely, $u$ computes a randomized sum-share version of $w_{u}$ by $w_{u}=\sum_{j=1}^{m} w_{u j}$, and sends the part $w_{u j}$ to every player $v \in Q_{j}$.

3. After player $u$ receives the parts $\left\{w_{v j}\right\}_{v \in U, Q, \ni u}$, she computes her share: $s_{u}(z) \leftarrow\left\{z_{j} \leftarrow \sum_{v \in U} w_{v j}\right\}_{Q_{\jmath} \ni u}$.

Lemma 13. Protocol Gen-MP computes correct Gen-SSS shares of $z=x \cdot y$.

Proof: Consider some quorum $Q_{k}$. As before, by the intersection property the members of $Q_{k}$ hold all the parts $\left\{z_{j}\right\}_{j=1}^{m}$, so they can compute the reconstruction function $\sum_{j=1}^{m} z_{j}$. Plugging in the expressions for $w_{u}$ and $w_{u}$ we have that

$$
\sum_{j=1}^{m} z_{j}=\sum_{j=1}^{m} \sum_{u \in U} w_{u j}=\sum_{u \in U} \sum_{j=1}^{m} w_{u j}=\sum_{u \in U} w_{u}=\sum_{u \in U} \sum_{i, j: \rho(i, j)=u} x_{i} y_{j},
$$

and since by the definition of $\rho$ exactly one player is in charge of computing every term $x_{i} y_{j}$, the last sum is equal to

$$
\sum_{i, j=1}^{m} x_{\imath} y_{j}=x \cdot y
$$

Theorem 14. Protocol Gen-MP computes $x \cdot y \mathcal{Q}$-privately. 
Proof: We wish to show that the information gained by $B$ is no different than if the input pieces were given to a trusted party who reconstructs $x$ and $y$ then shares $x y$. It suffices to provide a simulator that can construct a perfectly accurate view for $B$ using only the $z$-shares returned to $B$ by such a trusted party.

By definition there exists some quorum $Q \in \mathcal{Q}$ that is untainted, namely $Q \cap B=\emptyset$. Note, incidentally, that the trivial case in which $Q$ is of size 1 is easily dealt with - the single, uncorrupted party behaves as a trusted monarch and the protocol collapses, securely.

Let $\beta(B)=\left\{k: Q_{k} \cap B \neq \emptyset\right\}$ be the collection of (indices of) tainted quorums. Consider an $n \times m$ table of all the $w_{u j}$ values appearing in the protocol, whose row sums correspond to the players' $w_{u}$ values and whose column sums correspond to the final $z_{j}$ parts. Define the compromised region in this table to be

$$
W_{B}=\{(u, j) \mid u \in B\} \cup\{(u, j) \mid j \in \beta(B)\} .
$$

Along with the compromised values $\left\{w_{u}\right\}_{u \in B}, W_{B}$ indexes the values that $B$ is permitted to observe (within dishonest players or as received from good ones) in the multiplication protocol. The remaining region of $\left\{w_{u j}\right\}$, namely the values indexed by $\bar{W}_{B}$, is not included in $B$ 's view, nor are $\left\{w_{u}\right\}_{u \notin B}$.

We describe how to simulate an execution and then show that the fake view can be modified as needed when constraints on $x, y$ or $x y$ are made.

(1) Using the partial inputs $\left\{x_{\jmath}, y_{j}\right\}_{j \in \beta(B)}$, we first calculate $\left\{w_{u}\right\}_{u \in B}$ directly. We run "bad" players in $B$ honestly on these values, deriving a perfectly accurate distribution for values $\left\{w_{u}\right\}$ for all $u \in B$ and all $j$. The remaining compromised region lies in rows corresponding to uncompromised players. When the $\left\{z_{j}\right\}_{j \in \beta(B)}$ values are obtained from the imaginary trusted host, this compromised region is generated uniformly at random, subject to column constraints: $\sum_{u \in U} w_{u j}=z_{j}$.

(2) In an actual execution, the values of $\left\{w_{u}\right\}_{u \notin B}$ are determined by the input shares, then give rise to the sum-shares $\left\{w_{u j}\right\}$, which $B$ then partially observes (i.e., those in $W_{B}$ ). The values of $\left\{w_{u}\right\}_{u \in B}$ are likewise determined by input shares, but also revealed to $B$. For $j \in \beta(B)$, the column sums are determined by values revealed to $B$; for $j \notin \beta(B)$, the column sums include the uncompromised region, which is chosen uniformly at random (subject to row sums matching the uncompromised $\left.\left\{w_{u}\right\}_{u \notin B}\right)$.

Observe ("notably") that the second stage of experiment (1) is identical to setting arbitrary values in $\left\{w_{u}\right\}_{u \notin B}$ and then choosing the compromised region uniformly at random subject to row and column constraints. By the properties of sum-sharing, this in turn is equivalent to setting parts $x_{2}$ and $y_{i}$ for $i \notin \beta(B)$ to arbitrary values, executing honest programs on the results, and reporting only the compromised portion $\left\{w_{u j}\right\}_{u \notin B, j \in \beta(B)}$. (The $B$-view for rows in $B$ is clearly identical in both cases.)

Thus, experiments (1) and (2) provide identical distributions. As long as there exist appropriate settings to the uncompromised values, the "notable" observation (previous paragraph) that shows the distributions are identical continues to hold. Further, burdensome inspection shows that such settings always remain 
possible, even when $B$ learns further information, as when the final output value is revealed or when gates are tied together in composition. (Indeed, $B$ may be able to calculate some secrets and uncompromised entries along the way, even though it does not see them directly. The important point is that such entries can always be found when facing the simulated views.)

\subsection{Communication complexity}

Proposition 15. Denote the average quorum size by $q=\frac{1}{m} \sum_{j=1}^{m}\left|Q_{j}\right|$. Then protocol Gen-MP sends nmq messages.

This and several other propositions are proved in the Appendix.

Corollary 16. The communication complexity of protocol Gen-MP is $O\left(n^{2} m\right)$.

Our protocol performs quite favorably in comparison to the general construction of Hirt and Maurer [HM97], whose communication complexity appears to be $\Omega\left(m^{2.709}\right)$.

To see this, observe that [HM97] involves a recursive construction in which a set of players is replaced by three overlapping sets of players, each with size $2 / 3$ of the original. The depth $h$ of the recursion is thus determined by $(2 / 3)^{h} m \leq$ 3 , which gives $h \approx-\log _{3} m /\left(\log _{3} 2 / 3\right)>2.709 \log _{3} m$. The size (and hence communication complexity) thus exceeds $3^{h}=\Omega\left(m^{2.709}\right)$.

Because $m$ grows with the number of coalitions and is therefore generally exponential in the number of players, it is of far greater importance to reduce dependence on $m$. Our protocol incurs only a linear factor of $m$, with a small polynomial term in $n$.

\section{Efficient protocols for particular sharing schemes}

\subsection{The crumbling wall protocol}

The Crumbling Walls (CW) are a family of quorum systems due to [PW97]. This family includes, among others, the CWlog system (see Figure 1). The players in a wall are logically arranged in rows of varying widths. A quorum in a wall is the union of one full row and a representative from every row below the full row. The best crumbling walls are those in which the top row has width $n_{1}=1$ and every other row has width $n_{i} \geq 2$. In [PW97] it is shown that such walls are non-dominated coteries, i.e., their quorum access specification is unambiguous (recall Definition 6). Note that many of the quorums in a crumbling wall are small minorities - in the wall depicted in Figure 1 the smallest quorums are of size $O(\log n)$.

The CW-SSS secret sharing scheme Our multi-party protocol is based on the following secret sharing scheme, called CW-SSS, due to [NW96]. Consider a wall CW of $d$ rows, with row 1 having width $n_{1}=1$ and $n_{\imath} \geq 2$ for all $i \geq 2$. The basic secret unit $s$ is a single bit, therefore all the arithmetic in this section is over $G F(2)$. This secret $s$ is first randomly split into $d$ bits such that $a_{1}+\cdots+a_{d}=s$. Using these $a_{2}$ bits we can define their partial parities, $t_{i} \leftarrow a_{1}+\cdots+a_{i-1}$, and 




Fig. 1. A CWlog with $n=49$ elements and $d=15$ rows, with one quorum shaded. In this system row $i$ has width $\lfloor\log 2 i\rfloor$.

$t_{1}=0$. For a row $i$, split $t_{i}$ randomly into $n_{i}$ bits $A_{i}^{j}$ such that $A_{i}^{1}+\cdots+A_{i}^{n_{2}}=t_{i}$. The share $s_{i}^{j}$ of the $j$ 'th element in row $i$ contains two bits: $a_{i}$ and $A_{i}^{j}$.

A quorum $Q$, which contains a full row $i$ and a representative in each row $k>i$ can reconstruct the secret bit $s$ from the shares $\left\{s_{i}^{j}\right\}$ generated by $s=$ $\left(\sum_{j=1}^{n_{i}} A_{i}^{j}\right)+\left(\sum_{k \geq i} a_{k}\right)$

The CW-MP protocol Let secret $x$ be shared using pieces $\left(a_{i}, A_{\imath}^{j}\right)$ and let secret $y$ be shared using $\left(b_{i}, B_{i}^{j}\right)$. Linear combinations $(z=\alpha x+\beta y)$ are trivial: each player $(i, j)$ performs the operation locally on the corresponding pieces: $\left(\alpha a_{i}+\beta b_{i}, \alpha A_{i}^{j}+\beta B_{i}^{j}\right)$.

Multiplication is somewhat more complex, but it can be achieved through appropriate application of sum-sharing. We use $(i, j)$ to denote the $j$ 'th player in row $i$.

1. Local multiplication: Player $(i, j)$ locally computes $e_{i}^{j} \leftarrow a_{i} B_{i}^{j}+b_{i} A_{i}^{j}$.

2. Randomization: Player $(i, j)$ in row $i \geq 2$ flips a random value $r_{i}^{j}$. He then 2a. sends $r_{i}^{j}$ to the top player $(1,1)$, and

2b. sends $e_{i}^{j}+r_{i}^{j}$ to all other players $(i, k)$ in his row.

3. Partial reconstruction:

3a. The top player $(1,1)$ sets $c_{1} \leftarrow a_{1} b_{1}-\sum_{i \geq 2} \sum_{j=1}^{n_{i}} r_{i}^{j}$.

3b. Player $(i, j)$ in row $i \geq 2$ sets $c_{i} \leftarrow a_{i} b_{2}+\sum_{j=1}^{n_{i}}\left(e_{i}^{j}+r_{i}^{j}\right)$.

It is not hard to verify that all the members of each row $i$ compute the same value $c_{i}$, and that $\sum_{i=1}^{d} c_{i}=x y$. We now want to share the progressive parities of the $c_{i}$ 's in the successive rows.

4. Sharing across rows below: In each row $i=1, \ldots, d-1$ player $(i, 1)$ sumshares $c_{i}$ into $c_{i}=\sum_{j=1}^{n_{k}} t(i, k, j)$ for every row $k=i+1, \ldots, d$ and sends the share $t(i, k, j)$ to player $(k, j)$. 
5. Computing parity of rows above: Player $(i, j)$ in row $i \geq 2$ sets $C_{i}^{j} \leftarrow$ $\sum_{k=1}^{i-1} t(i, k, j)$. Thereby each row $i$ has secretly computed (without reconstruction) the sum-share of the partial parity $c_{1}+\cdots+c_{i-1}$.

The communication complexity of this protocol is clearly $O\left(n^{2}\right)$ bits and $O(1)$ rounds of message exchange.

Proposition 17. The preceding protocols securely compute shares of $z=\alpha x+\beta y$ (resp., $z=x \cdot y$ ) $\mathcal{Q}$-privately according to the CW-SSS scheme.

\subsection{The finite projective plane protocol}

In this section we describe an efficient $O\left(n^{2}\right)$ protocol for the case where the underlying quorum system is a finite projective plane (FPP) [Mae85]. For a prime $r$ let $t=r^{k}$ for some integer $k$. Then the finite projective plane of order $t$ is a quorum system with $n=t^{2}+t+1$ players and $m=t^{2}+t+1$ quorums of size $t+1$. Moreover, each player is a member of $t+1$ quorums, and the intersection of every two quorums consists of a single player.

Note that since $n=m$ and the average quorum size $q \approx \sqrt{n}$ the general protocol of section 3 already has a polynomial message complexity of $O\left(n^{2.5}\right)$ for the FPP system. However, here we can use a much more efficient secret sharing scheme: The size of the share held by each player is 1 or 2 , in comparison to the $O(m)$-sized shares used in Gen-SSS. Thus the local computations in the FPP-MP protocol take constant time.

The FPP-SSS secret sharing scheme In this section all the arithmetic is performed in the ring $\mathbf{Z}_{t}$, where $t$ is the order of the finite projective plane. Let $x$ be the secret to be shared. As in Gen-SSS, $x$ is represented randomly as a sum-share $x=\sum_{j=1}^{m} x_{j} \quad(\bmod t)$. Then the share of player $u$ is the sum of the parts corresponding to the quorums that contain $u$ :

$$
a_{u}(x) \leftarrow \sum_{Q, \ni u} x_{j}(\bmod t) .
$$

Proposition 18. FPP-SSS is a secret sharing scheme realizing the quorum access specification $(\mathcal{G}(F P P), \mathcal{B}(F P P))$.

The FPP-MP protocol Assume that $x$ and $y$ are shared among the players according to the FPP-SSS scheme. In order to compute $z=\alpha x+\beta y \mathcal{Q}$-privately the players locally compute $a_{u}(z) \leftarrow \alpha a_{u}(x)+\beta a_{u}(y)$. It is clear that the resultant shares are valid and no information was revealed by this computation.

To compute the product $x y$, each player $u$ performs the following steps:

1. Player $u$ locally computes the product $w_{u} \leftarrow a_{u}(x) \cdot a_{u}(y)$.

2. Player $u$ secret-shares the value $w_{u}$ among all the players using the FPPSSS scheme. Namely, $u$ computes a randomized sum-share version of $w_{u}$ by $w_{u}=\sum_{j=1}^{m} w_{u j}$. Then for every $v \in U u$ computes $\alpha_{u v}=\sum_{Q_{j} \ni v} w_{u j}$ and sends $\alpha_{u v}$ to $v$. 
3. After player $u$ receives all the values $\left\{\alpha_{v u}\right\}_{v \in U}$, she computes her share $a_{u}(z)$ as $a_{u}(z) \leftarrow \sum_{v \in U} \alpha_{v u}$.

Lemma 19. Protocol FPP-MP computes correct FPP-SSS shares of $z=x \cdot y$.

Proposition 20. The preceding protocols securely compute shares of $z=\alpha x+\beta y$ (resp., $z=x \cdot y$ ) $\mathcal{Q}$-privately according to the FPP-SSS scheme.

Remark: In the above scheme the arithmetic modulus $t$ is tied to the system size $n$ since $n=t^{2}+t+1$. To disconnect this tie, we devised a variant of the FPPSSS scheme in which the modulus can be arbitrary (e.g., we can use $G F(2)$ ). The price we pay for this extra freedom is that the share held by every player will include an additional correcting term. We omit the details in this abstract.

\subsection{Hierarchical quorum protocols}

In the hierarchical quorum system (HQS), due to [Kum91], the individuals are the leaves of a complete ternary tree in which internal nodes are 2-of-3 majority gates. The related Tree quorum system [AE91] employs full ternary trees, but the center child of each tree is a leaf. The HQS and Tree systems enjoy high availability and low load.

The HQS-SSS secret sharing scheme A secret sharing scheme for HQS appears in [NW96]; here we give only a brief description of the construction, which is essentially recursive Shamir sharing.

If $p$ is a node in a ternary tree, let $p .1, p .2$ and $p .3$ be its children (if any). This gives a natural labeling for nodes, e.g. "root.1.3.1.2." Let $\operatorname{split}(x)$ be a distribution over $\mathrm{GF}(4)^{3}$ obtained by selecting a random $a$ and computing $(f(01), f(10), f(11))$ where $f(u)=a u+x$.

Sharing value $v$ for secret $x$ is straightforward recursion from the root: Share $(x, v, p)$ sets $x[p] \leftarrow v$ if $p$ is a leaf; otherwise set $\left(v_{1}, v_{2}, v_{3}\right) \leftarrow \operatorname{split}(v)$, then recurse on $\operatorname{Share}\left(x, v_{\imath}, p . i\right)(i=1,2,3)$.

The HQ-MP protocol Let secret $x$ be shared using pieces $x[p]$ and let secret $y$ be shared using $y[p]$. To perform a linear combination $(z=\alpha x+\beta y)$, players simply perform the operation locally: $z[p] \leftarrow \alpha x[p]+\beta y[p]$.

Although intuitively obvious, a recursive construction for multiplication using [BGW88, CCD88] requires some care. In particular, the internal nodes are "virtual," namely represented by sets of players; thus otherwise obvious steps such as " $p$ sends $m$ to $q$ " are ill-defined when $p$ and $q$ are not leaf nodes.

The steps needed to apply [BGW88, CCD88] include linear combination and multiplication of local values, and sending messages. (Sharing, reconstruction, selecting random values, and degree reduction can be built with these primitives.) It remains to be seen, therefore, how messages are sent from $p$ to $q$.

There are four cases. If $p$ and $q$ are leaves, $m$ is sent directly. If $p$ is a leaf while $q$ is internal, $p$ runs Share $\left(m_{p q}, m, q\right) ; m_{p q}$ is the label for the secret message, held by players under $q$. If $p$ is internal while $q$ is a leaf, players under $p$ send their 
shares of $m$ to $q$ for reconstruction. If $p$ and $q$ are internal, then $p . i$ recursively "sends" its share of $m$ to $q . i$ (for $i=1,2,3$ ).

The communication cost of the overall construction is $O\left(n^{2}\right)$ per gate. Because [BGW88, CCD88] can be executed with one multiplication per virtual player, the primary concern is to measure the cost of "sending" messages. Straightforward arguments show that the worst case trees are those that are least balanced (e.g., all the subtrees lie under left children). In this extreme, the cost of sending a message at height $H$ from the bottom is $\Omega(H n)$. Multiplication requires $O(1)$ simulated messages for any particular troika; thus the net cost of multiplication is $\mathrm{O}\left(n^{2}\right)$.

Remark: This protocol can be seen as a particular instance of the [HM97] construction, if certain unconstrained choices are cleverly made; thus, a security argument can be derived from [HM97, BGW88, CCD88]. Note, however, that the constructive argument in [HM97] permits several degrees of freedom, most of which lead to an exponential algorithm for these quorum systems.

\section{Acknowledgments}

We are very grateful to Daniel Bleichenbacher for many highly useful discussions, and for introducing the result of Hirt and Maurer to us. We also thank Martin Hirt and Ueli Maurer for discussions and comparisons of their work.

\section{References}

[AE91] D. Agrawal and A. El-Abbadi. An efficient and fault-tolerant solution for distributed mutual exclusion. ACM Trans. Comp. Sys., 9(1):1-20, 1991.

[Bea91a] D. Beaver. Foundations of secure interactive computing. In Advances in Cryptology - CRYPTO'91, LNCS 576, pages 377-391. Springer-Verlag, 1991.

[Bea91b] D. Beaver. Secure multiparty protocols and zero-knowledge proof systems tolerating a faulty minority. J. Cryptology, 4(2):75-122, 1991.

[BG89] D. Beaver and S. Goldwasser. Multiparty computation with faulty majority. In Proc. 30th IEEE Symp. Foundations of Comp. Sci. (FOCS), pages 468473, 1989.

[BH92] D. Beaver and S. Haber. Cryptographic protocols provably secure against dynamic adversaries. In Advances in Cryptology - EUROCRYPT'92, LNCS 658, pages 307-323. Springer-Verlag, 1992.

[BGW88] M. Ben-Or, S. Goldwasser, and A. Wigderson. Completeness theorems for non-cryptographic fault-tolerant distributed computation. In Proc. 20th ACM Symp. Theory of Computing (STOC), pages 1-10, Chicago, Illinois, 2-4 May 1988.

[BKK94] S. Ben-David, M. Karchmer, and E. Kushilevitz. On ultrafilters and NP. In Proceedings of the 9th Annual Conference on Structure in Complexity Theory, pages 97-105. IEEE Computer Society Press, 1994.

[BL88] J. Benaloh and J. Leichter. Generalized secret sharing and monotone functions. In Advances in Cryptology - CRYPTO'88, LNCS 403, pages 27-36. Springer-Verlag, 1988.

[Bla79] G. R. Blakely. Safeguarding cryptographic keys. Proc. AFIPS, NCC, 48:313-317, 1979. 
[CCD88] D. Chaum, C. Crépeau, and I. Damgård. Multiparty unconditionally secure protocols. In Proc. 20th ACM Symp. Theory of Computing (STOC), pages 11-19, Chicago, Illinois, 2-4 May 1988.

[CFGN96] R. Canetti, U. Feige, O. Goldreich, and M. Naor. Adaptively secure multiparty computation. In Proc. 28th ACM Symp. Theory of Computing (STOC), pages 639-648, 1996.

[CK91] B. Chor and E. Kushilevitz. A zero-one law for Boolean privacy. SIAM J. Discrete Math., 4:36-47, 1991.

[DDFY94] A. De Santis, Y. Desmet, Y. Frankel, and M. Yung. How to share a function securely. In Proc. 26th ACM Symp. Theory of Computing (STOC), pages 522-533, 1994.

[DGS85] S. B. Davidson, H. Garcia-Molina, and D. Skeen. Consistency in partitioned networks. ACM Computing Surveys, 17(3):341-370, 1985.

[Für88] Z. Füredi. Matchings and covers in hypergraphs. Graphs and Combinatorics, 4:115-206, 1988.

[GB85] H. Garcia-Molina and D. Barbara. How to assign votes in a distributed system. J. $A C M, 32(4): 841-860,1985$.

[GHY88] Z. Galil, S. Haber, and M. Yung. Cryptographic computation: Secure faulttolerant protocols and the public-key model. In Advances in Cryptology CRYPTO'87, LNCS 293, pages 135-155. Springer-Verlag, 1988.

[GMR89] S. Goldwasser, S. Micali, and C. Rackoff. The knowledge complexity of interactive proof systems. SIAM J. Computing, 18(1):186-208, 1989.

[GMW86] O. Goldreich, S. Micali, and A. Wigderson. Proofs that yield nothing but their validity and a methodology of cryptographic protocol design. In Proc. 27th IEEE Symp. Foundations of Comp. Sci. (FOCS), pages 174-187. IEEE, 1986.

[GMW87] O. Goldreich, S. Micali, and A. Wigderson. How to play any mental game, or a completeness theorem for protocols with honest majority. In Proc. 19th ACM Symp. Theory of Computing (STOC), pages 218-229, 1987.

[Her84] M. P. Herlihy. Replication Methods for Abstract Data Types. PhD thesis, Massachusetts Institute of Technology, MIT/LCS/TR-319, 1984.

[HM97] M. Hirt and U. Maurer. Complete characterization of adversaries tolerable in secure multi-party computation. In Proc. 16th ACM Symp. Princip. of Dist. Comp. (PODC), pages 25-34, August 1997.

[IK93] T. Ibaraki and T. Kameda. A theory of coteries: Mutual exclusion in distributed systems. IEEE Trans. Par. Dist. Sys., 4(7):779-794, 1993.

[ISN87] M. Ito, A. Saito, and T. Nishizeki. Secret sharing schemes realizing general access structure. In Proc. IEEE Global Telecommunication Conf. (Globecomm 87), pages 99-102, 1987.

[Kum91] A. Kumar. Hierarchical quorum consensus: A new algorithm for managing replicated data. IEEE Trans. Comput., 40(9):996-1004, 1991.

[Mae85] M. Maekawa. A $\sqrt{n}$ algorithm for mutual exclusion in decentralized systems. ACM Trans. Comp. Sys., 3(2):145-159, 1985.

[MR91] S. Micali and P. Rogaway. Secure computation. In Advances in Cryptology - CRYPTO'91, LNCS 576, pages 392-404. Springer-Verlag, 1991.

[MV88] S. J. Mullender and P. M. B. Vitányi. Distributed match-making. Algorithmica, 3:367-391, 1988.

[NW94] M. Naor and A. Wool. The load, capacity and availability of quorum systems. In Proc. 35th IEEE Symp. Foundations of Comp. Sci. (FOCS), pages 214-225, 1994. To appear in SIAM J. Computing 1998. 
[NW96] M. Naor and A. Wool. Access control and signatures via quorum secret sharing. In Proc. 3rd ACM Conf. Comp. and Comm. Security, pages 157168, New Delhi, India, 1996. Also available as Theory of Cryptography Library record 96-08, http://theory.lcs.mit.edu/ tcryptol/1996.html.

[Owe82] G. Owen. Game Theory. Academic Press, second edition, 1982.

[PW97] D. Peleg and A. Wool. Crumbling walls: A class of practical and efficient quorum systems. Distributed Computing, 10(2):87-98, 1997.

[RB89] T. Rabin and M. Ben-Or. Verifiable secret sharing and multiparty protocols with honest majority. In Proc. 21st ACM Symp. Theory of Computing $(S T O C)$, pages $73-85,1989$.

[Sha79] A. Shamir. How to share a secret. Comm. ACM, 22(11):612-613, 1979.

\section{Appendix}

Proof of Proposition 15: The only messages sent by the protocol are the shares $w_{u j}$ sent in step 2. Each player $u$ sends the part $w_{u j}$ to every player $v \in Q_{j}$, giving: $n \sum_{j=1}^{m}\left|Q_{j}\right|=n m q$.

Proof of Proposition 18: The reconstruction function for a quorum $Q_{3}$ is the sum of all the shares held be the members of $Q_{j}$. From the definition of the shares, we know: $\sum_{u \in Q_{j}} a_{u}(x)=\sum_{u \in Q_{j}} \sum_{Q_{\imath} \ni u} x_{\imath}$. Since every two quorums intersect in a single player, the part $x_{i}$ appears once in the double summation for every $i \neq j$. The part $x_{j}$ itself appears $t+1$ times, but since the arithmetic is mod $t$, the last sum equals $x$. Hence every quorum $Q_{j}$ can reconstruct $x$.

Now consider a set $B \in \mathcal{B}($ FPP $)$. Then by definition there exists some quorum $Q_{i} \in$ FPP such that $Q_{i} \subseteq U \backslash B$. The part $x_{\imath}$ appears in the sum $a_{u}(x)$ only for $u \in Q_{i}$. Therefore $x_{i}$ is independent of the shares held by members of $B$. From this it is easy to see that the union of the shares of all players in $B$ gives no information about $x$.

Proof of Lemma 19: First we show that the $w_{u}$ 's sum up to $z$.

$$
\sum_{u \in U} a_{u}(x) a_{u}(y)=\sum_{u \in U}\left(\sum_{Q_{\imath} \ni u} x_{i}\right)\left(\sum_{Q_{j} \ni u} y_{j}\right)=\sum_{u \in U} \sum_{\substack{Q_{i} \ni u \\ Q_{j} \ni u}} x_{i} y_{j} .
$$

The term $x_{i} y_{j}$ appears in the sum once for every $u \in Q_{i} \cap Q_{j}$. Therefore $x_{i} y_{j}$ appears once when $i \neq j$ and $t+1$ times when $i=j$. Since we are working $\bmod t$ we conclude that $\sum_{u \in U} a_{u}(x) a_{u}(y)=\sum_{i, j=1}^{m} x_{i} y_{j}=x y$. Now consider some quorum $Q_{i}$. Plugging $a_{v}(z)$ into the reconstruction function for $Q_{i}$ we get

$$
\sum_{v \in Q_{i}} a_{v}(z)=\sum_{v \in Q_{2}} \sum_{u \in U} \alpha_{u v}=\sum_{v \in Q_{2}} \sum_{u \in U} \sum_{Q_{\jmath} \ni v} w_{u \jmath}=\sum_{u \in U} \sum_{v \in Q_{2}} \sum_{Q_{j} \ni v} w_{u j} .
$$

Now we use a similar trick (this time holding $Q_{i}$ fixed). For any $u$, the part $w_{u j}$ appears in the sum once for every $v \in Q_{i} \cap Q_{j}$. Since we are working $\bmod t$,

$$
\sum_{u \in U} \sum_{j=1}^{m} w_{u j}=\sum_{u \in U} w_{u}=\sum_{u \in U} a_{u}(x) a_{u}(y)=x y
$$

